\title{
In silico prediction of telomerase reverse transcriptase inhibitors using modified retinol for the treatment of arsenical cancer
}

\author{
Kipchu Tshering and Mir Misbahuddin
}

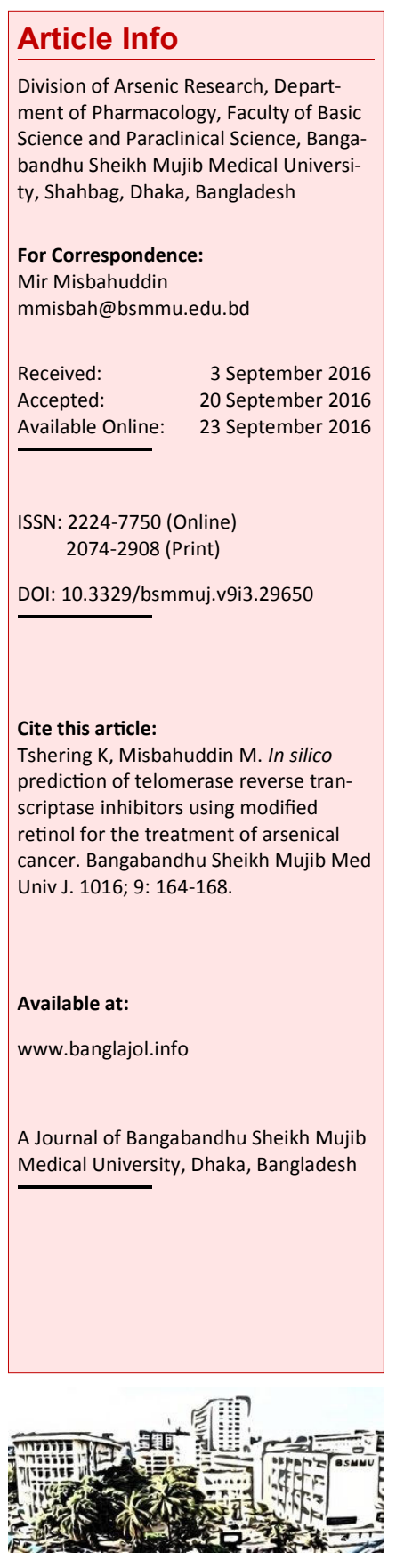

\section{Abstract}

Telomerase activity is expressed in the cancerous conditions which can be the target for anticancer activity by inhibiting telomerase reverse transcriptase enzyme. In this in silico study, retinol molecule was modified to predict the inhibitors of telomerase reverse transcriptase for the treatment of arsenical cancer. The inhibitors were predicted through molecular docking of modified retinol with telomerase reverse transcriptase. Taking into account of low binding energy and high binding affinity, two new compounds (\#606 and \#609) were predicted as the inhibitors of telomerase reverse transcriptase. The prediction of these two compounds was further supported by drug-likeness test through administration, distribution, metabolism, excretion and toxicity evaluation. The compounds were also compared with some of the known inhibitors of telomerase reverse transcriptase.

\section{Introduction}

Arsenicosis develops following chronic consumption of high concentration of arsenic through drinking water.1 Consumption of water contaminated with arsenic for a longer period can result in cancer of skin and other internal organs like liver, kidney, bladder, lungs, etc.? In Bangladesh, it is estimated that nearly 80 million people are affected by arsenic poisoning and one in ten has probability of developing cancer from the poisoning. 3

Unfortunately, till today, there is no specific treatment for arsenical cancer. Telomerase activity is expressed in cancerous conditions including arsenical cancer. $\underline{4}$ Inhibitors of telomerase reverse transcriptase enzyme may be presumed to be effective in arsenical cancer. Identification of such a molecule can be predicted from molecular docking since docking process is used to understand the drug-receptor interaction and now-a-days plays an important role in modern drug designing.

This study was aimed for the prediction of inhibitors of telomerase reverse transcriptase as specific molecules for the treatment of arsenical cancer through computer simulation. Inhibitors of telomerase reverse transcriptase enzyme were predicted from modified retinol molecule through molecular docking and virtual screening.

\section{Materials and Methods}

This in silico study was done using different software like AutoDock 4, AutoDock Vina, Discovery Studio, $\underline{5}$ Marvin Sketch and FAFDrug3. The main work consists of the molecular docking of modified retinol molecule with telomerase reverse transcriptase and analyzing the docking results in terms of binding energy.

Downloading the structures of retinol and telomerase reverse transcriptase

Both the retinol molecule and telomerase reverse transcriptase were downloaded from the worldwide protein data bank website (http://www.rcsb.org/pdb/home/home.do) and purified from other heterogeneous structures to get the pure structure of retinol and telomerase reverse transcriptase. Discovery Studio software was used for the purification of the structures.

\section{Modification of retinol molecule as new ligand}

Retinol molecule was modified in its structure by adding, deleting and substituting different atoms in its structure and by adding different functional groups to its structure for the molecular docking of modified retinol with telomerase reverse transcriptase. The modification of retinol structure was done in Discovery Studio. 


\section{Molecular Docking}

Docking process was done in AutoDock트 and AutoDock Vina. 7 Modified retinol molecules were docked with the telomerase reverse transcriptase structure in AutoDock Vina and the binding energies were recorded. The modified compound with least binding energy was further docked in AutoDock 4 to view the molecular interaction.

\section{Naming of modified compounds}

All the new compounds from retinol were named as per International Union of Pure and Applied Chemistry (IUPAC) naming system. MarvinSketch, a Java based chemical drawing tool, was used for naming of the molecules.

\section{Drug-likeness evaluation}

The selected compounds with least binding energy after molecular docking were evaluated for ADMETox (Absorption, Distribution, Metabolism, Excretion and Toxicity) concerning the druglikeness properties through FAF-Drug3 web server. 8

\section{Comparison with known telomerase reverse transcriptase inhibitors}

Molecular docking of some known inhibitors of telomerase reverse transcriptase was done with the telomerase reverse transcriptase and the binding

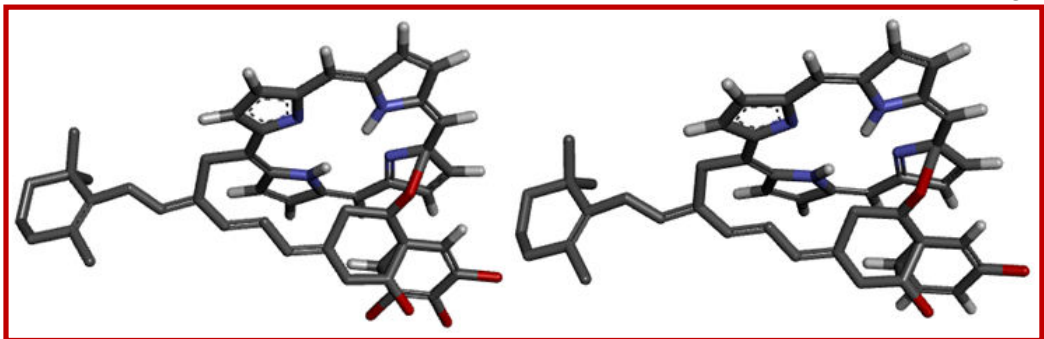

Figure 1: Chemical structure of compound 606 (left) and compound 609 (right) energies were compared with the binding energy of predicted new compounds from this study.

\section{Results}

Retinol molecule was structurally modified into 400 different structures by deleting/shortening side chain and adding ethyl, methyl, imidazolyl, acetyl, amino, thiol group. Fourteen chemical compounds showed binding energy of less than $-5.0 \mathrm{kcal} / \mathrm{mol}$ with telomerase reverse transcriptase. Further modification of these 14 compounds by adding different functional groups resulted in the formation of another 420 compounds. Analyzing the binding energy of 420 new compounds with telomerase reverse transcriptase, two compounds (\#606 and \#609) were found to have the minimum binding energy $(-7.8 \mathrm{kcal} / \mathrm{mol})$. The chemical structure of these two compounds is shown in Figure 1.

IUPAC name was assigned to all the compounds. Compound \#606 with IUPAC name 21,22,23,24tetraazapentacyclo[16.2.1.1 $\left.1^{3},{ }^{6} .1^{8},{ }^{11} .1^{13},{ }^{16}\right]$ tetracosa1,3,5,7,9,11(23),12,14,16,18(21),19-undecaene; 3,4,5trihydroxybenzaldehyde; 3,7-dimethyl-9-(2, 6,6trimethylcyclohexa-1,4-dien-1-yl)nonan-1-ol and compound \#609 with IUPAC name 21,22,23,24tetraazapentacyclo[16.2.1.1 $\left.{ }^{3}, 6.1^{8},{ }^{11} .1^{13},{ }^{16}\right]$ tetracosa1,3,5,7,9,11(23),12,14,16,18(21),19-undecaene; 3,7dimethyl-9-(2,6,6-trimethylcyclo-hexa-1,4-dien-1-yl) nonan-1-ol; 3-hydroxybenzal-dehyde were predicted as the inhibitors of telo-merase reverse transcriptase since both the compounds had less binding energy of $-7.8 \mathrm{kcal} / \mathrm{mol}$ each.

Molecular interaction between the selected compounds and telomerase reverse transcriptase was viewed in AutoDock4. Compound 606 and 609

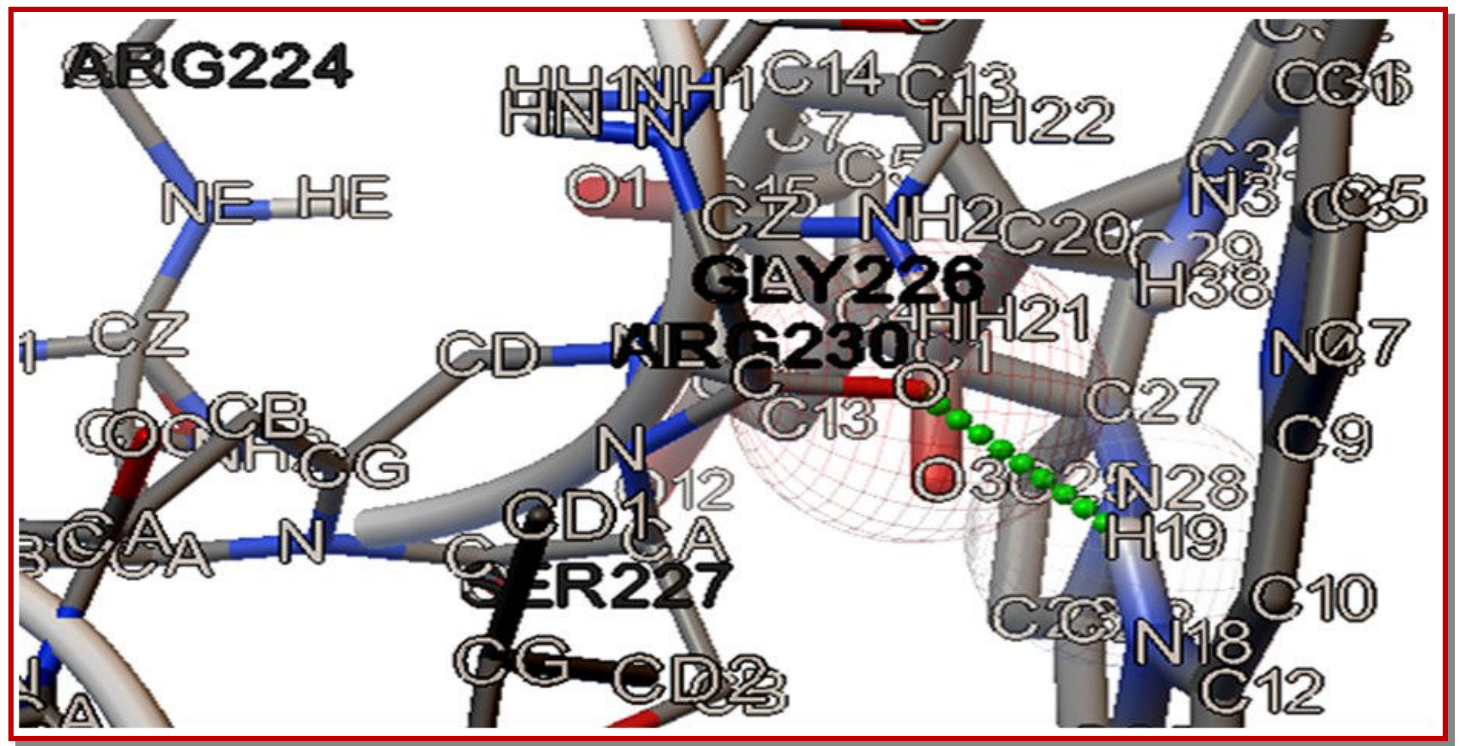

Figure 2: Interaction of compound 606 with telomerase reverse transcriptase (H-bond shown by green spheres) 


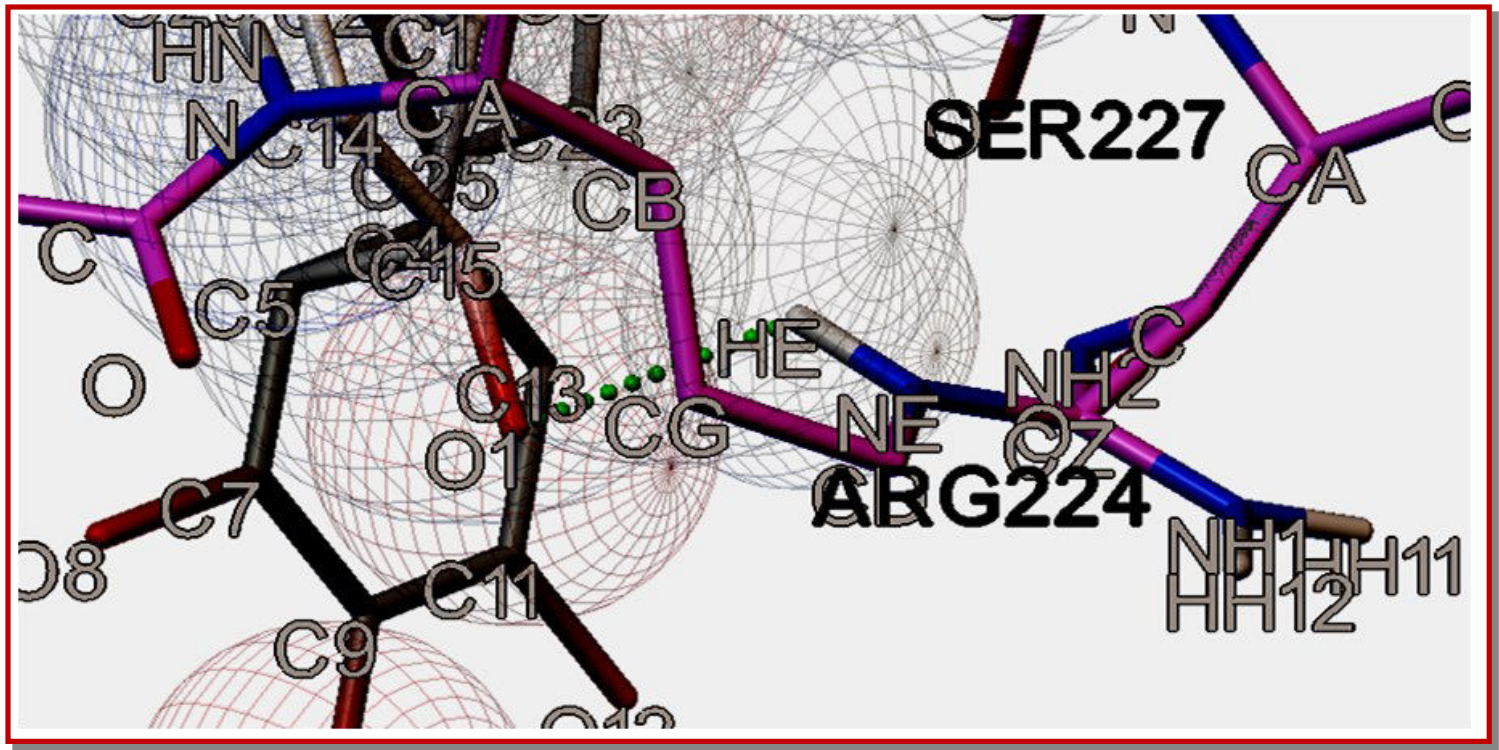

Figure 3: Interaction of compound 609 with telomerase reverse transcriptase (H-bond shown by green spheres)

Table I

Binding energy of some known inhibitors, compound \#606 and \#609 with telomerase reverse transcriptase protein

Compounds Binding energy

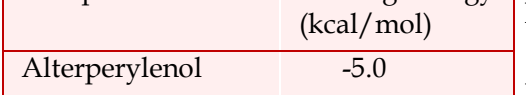

Boldine $\quad-5.7$

Crocin $\quad-5.3$

Curcumin $\quad-4.6$

Dideoxyguanosine $\quad-3.7$

triphosphate

Dimethyl sulfoxide $\quad-2.1$

Epigallocatechin $\quad-4.7$

Genistein $\quad-4.5$

Harmine $\quad-4.8$

Helenalin $\quad-5.5$

Helenin $\quad-4.9$

Isoprenoid $\quad-4.0$

\begin{tabular}{ll} 
Levofloxacin $\quad-4.8$ \\
\hline
\end{tabular}

Ofloxacin $\quad-4.7$

Pristimerin $\quad-0.7$

Tanshinone $\quad-6.1$

Tretinoin $\quad-4.7$

Tocotrienol $\quad-3.4$

Triethylene $\quad-2.6$

tetraamine

Zidovudine

$-4.1$

Compound 214

$-7.5$

Compound 606

$-7.8$

Compound 609

$-7.8$ interacted with telomerase reverse transcriptase protein by forming hydrogen bond between the atoms of the compounds and the atoms of the amino acids in telomerase reverse transcriptase protein. Compound 606 interacted with the enzyme by hydrogen bond between the H19 (hydrogen number 19) atom in porphyrin ring and $\mathbf{O}$ (oxygen) atom of the amino acid ARG230 (arginine number 230) of telomerase reverse transcriptase (Figure 2). The amino acids sequence number in telomerase reverse transcriptase was labeled as per the sequence number in that of the downloaded 4B18 protein, the crystal structure of human importin alpha 5 with telomerase reverse transcriptase NLS peptide.

Compound 609 interacted with the telomerase reverse transcriptase protein by hydrogen bond between O1 (oxygen number 1) atom and HE (epsilon hydrogen) atom of the amino acid ARG224 (arginine number 224) of telomerase reverse transcriptase (Figure 3). The amino acid sequence number mentioned here was as per the sequence number in the downloaded $4 \mathrm{~B} 18$ protein, the crystal structure of human importin alpha 5 with telomerase reverse transcriptase NLS peptide.

These two compounds were selected and predicted as the potential inhibitors which were further supported by the accepting the drug- likeness test through ADMETox evaluation and by having the minimum binding energy with telomerase reverse transcriptase when compared with some of the known inhibitors of telomerase reverse transcrip-tase (Table I).

\section{Discussion}

In this study, two compounds were synthesized from retinol in silico using different software those have inhibitory function on telomerase reverse transcriptase. These compounds were accepted through ADMETox evaluation. Compound 606 and 609 chemically composed of modified retinol molecule with added side chains of porphyrin and benzoyl rings with five oxygen atoms in compound 606 and three oxygen atoms in compound 609 .

No study has been done to predict new compounds as the inhibitors of telomerase reverse transcriptase for use in the treatment of arsenical cancer by modification of retinol molecule through molecular docking.

Retinol was used as a lead compound. Retinol, like a-tocopherol, is usually suggested with other antioxidant vitamins and minerals for the treatment of arsenical keratosis (non-malignant skin lesion). Previous study from this department had used atocopherol as lead compound and identified one compound (\#214). 9

Acyclic retinoid induces partial differentiation, down-regulates telomerase reverse transcriptase mRNA expression and telomerase activity, and induces apotosis in human hepatoma-derived cell lines. 10

Studies were done for different inhibitors of telomerase reverse transcriptase in other disease 
conditions like boldine, $\underline{11}$ crocin, $\underline{12}$ dimethyl sulfoxide, $\underline{13}$ harmine, $\underline{14}$ helenalin, $\underline{15}$ pristimerin, $\underline{16}$ tanshinone, 17 triethylene tetraamine, $\underline{18}$ and tocotrienol.19. Regarding the use of vitamin $\mathrm{A}$ in cancer, vitamin $\mathrm{A}$ has shown to prevent squamous cell carcinoma of skin significantly $\underline{\underline{20}}$ and all-trans retinoic acid has shown complete remission of acute promyelocytic leukemia with daily supplementation of $25,000 \mathrm{IU}$ in $95 \%$ of patients in study group. $\underline{21}$

The present authors compare the inhibitory effects of compound \#606, \#609 with 21 other inhibitors of telomerase reverse transcriptase using mole-cular docking. It is apparent that compound \#606 and \#609 are the most effective drugs. Even these are more potent than compound \#214 as inhibitor of telomerase reverse transcriptase. These three compound have similarities in having the porphyrin ring in their chemical structure. It can be assumed that porphyrin ring can be the functional group which facilitates the interaction of the new compounds with telomerase reverse transcriptase protein in regard to higher binding affinity and lower binding energy.

\section{Conclusion}

Two compounds (\#606 and \#609) are identified from retinol using in silico study which have potent inhibitor effect against telomerase reverse transcriptase. Future studies on the synthesis of these compound and their effectiveness in wet lab will confirm the present findings.

\section{References}

1. Misbahuddin M. Arsenicosis: A global issue. New York, Science Publishing Group, 2015, pp 1204.

2. Misbahuddin M (ed). Applied research on arsenic in Bangladesh. Dhaka, WHO and Directorate General of Health Services, GOB, 2007, pp 1-135.

3. Argos M, Kalra T, Rathouz TJ, Chen Y, Pierce B, Parvez F, Islam T, Amed A, Zaman MR, Hasan R, Sarwar G, Slavkovich V, Geen AV, Graziano J, Ahsan $\mathrm{H}$. Arsenic exposure from drinking water, and all-cause and chronic-disease mortalities in Bangladesh (HEALS): A prospective cohort study. Lancet 2010; 376: 252-58.

4. Mo J, Xia Y, Ning Z, Wade TJ, Mumford JL. Elevated human telomerase reverse transcriptase gene expression in blood cells associated with chronic arsenic exposure in inner Mongolia, China. Environmental Health Prospectives. 2009; 117: 354-60.
5. Accelrys Software Inc. Discovery Studio modeling environment, Release 4.0. San Diego. Accelrys Software Inc. 2013.

6. Morris GM, Huey R, Lindstrom W, Sanner M F, Belew RK, Goodsell DS, Olson AJ. AutoDock4 and AutoDockTools4: automated docking with selective receptor flexibility. J Comput Chem. 2009; 30: 2785-91.

7. Trott $\mathrm{O}$, Olson AJ. AutoDock Vina: Improving the speed and accuracy of docking with a new scoring function, efficient optimization and multithreading. J Comput Chem. 2010; 31: 455-61.

8. Lagorce D, Sperando O, Golans H, Miteva MA, Villoutrix BO. FAF-Drugs2: Free ADME/tox filtering tool to assist drug delivery and chemical biology projects. BMC Bioinformatics. 2008; 9.

9. Mustafa TG. In silico identification of a common biological target for the compounds that are suggestive in the treatment of arsenicosis and design of a drug that acts on it. MD thesis, Bangabandhu Sheikh Mujib Medical University, Dhaka, 2014.

10. Yasuda I, Shiratori $Y$, Adachi S, Obora A, Takemur M, Okuno M, Shidoji Y, Seishima M, Muto $\mathrm{Y}$, Moriwaki $\mathrm{H}$. Acyclic retinoid induces partial differentiation, down-regulates telomerase reverse transcriptase mRNA expression and telomerase activity, and induces apotosis in human hepatoma-derived cell lines. J Hepatol. 2002; 36: 660-71.

11. Noureini SK, Wink M. Dose-dependent cytotoxic effects of boldine in HepG-2 cells-telomerase inhibition and apoptosis induction. Molecules 2015; 20: 3730-43.

12. Noureini SK, Wink M. Antiproliferative effects of crocin in HepG-2 cells by telomerase inhibition and hTERT down-regulation. Asian Pacific J Cancer Prev. 2012; 13: 2305-09.

13. Sharma RA, Gescher AJ, O'Byrne KJ, Steward WP. Familiar drugs may prevent cancer. Postgrad Med J. 2001; 77: 492-97.

14. Zhao L, Wink M. The $\beta$-carboline alkaloid harmine inhibits telomerase activity of MCF-7 cells by down-regulating hTERT mRNA expression accompanied by an accelerated senescent phenotype. Peer J. 2013; 1: 174.

15. Zhang $\mathrm{Z}, \mathrm{Xu} \mathrm{L}$, Cheung HY. The inhibitory effect of helenalin on telomerase activity is attributed to the alkylation of the CYS445 residue: Evidence from QM/MM simulations. J Mol Graph Model. 2014; 51: 97-103.

16. Deeb D, Gao X, Liu Y, Pindolia K, Gautam SC. Inhibition of hTERT/telomerase contributes to the antitumor activity of pristimerin in pancreatic ductal adenocarcinoma cells. Oncol Rep. 2015; 34: 518-24. 
17. Soares J, Wang X, Lee KH, Jarstfer_MB. Orthoquinone tanshinones directly inhibit telomerase through an oxidative mechanism mediated by hydrogen peroxide. Bioorg Med Chem Lett. 2011; 21: 7474-78.

18. Yin F, Liu J, Peng X. Triethylene tetraamine: A novel telomerase inhibitor. Bioorg Med Chem Lett. 2003; 13: 3923-26.

19. Eitsuka T, Nakagawa K, Miyazawa T. Downregulation of telomerase activity in DLD-1 human colorectal adenocarcinoma cells by tocotrienol.
Biochem Biophys Res Commun. 2006; 348: 170-75.

20. Moon TE, Levine N, Cartmel B, Bangert JL, Rodney S, Dong Q, Peng YM, Alberts DS. Effect of retinol in preventing squamous cell skin cancer in moderate-risk subjects: A randomised doubleblind controlled trial. Cancer Epidemiol Biomark Prev. 1997; 6: 949-96.

21. Huang ME, Ye YC, Chen SR, Chai JR, Lu JX, Zhoa L, Gu LJ, Wang ZY. Use of all-trans retinoic acid in the treatment of acute promyelocytic leukaemia. Blood 1988; 72: 567-72. 